\title{
Workforce Effects and the Evolution of Complex Sociality in Wild Damaraland Mole Rats
}

\author{
Andrew J. Young, ${ }^{1, *}$ Jennifer U. M. Jarvis, ${ }^{2}$ James Barnaville, ${ }^{1}$ and Nigel C. Bennett ${ }^{3}$ \\ 1. Centre for Ecology and Conservation, School of Biosciences, University of Exeter, Penryn, Cornwall TR10 9FE, United Kingdom; \\ 2. Department of Biological Sciences, University of Cape Town, Rondebosch 7701, South Africa; 3. Department of Zoology and \\ Entomology, University of Pretoria, Pretoria 0002, South Africa
}

Submitted January 10, 2015; Accepted March 5, 2015; Electronically published June 1, 2015

Dryad data: http://dx.doi.org/10.5061/dryad.1366f.

ABSTRACT: Explaining the evolution of eusocial and cooperatively breeding societies demands that we understand the effects of workforce size on the reproductive success of breeders. This challenge has yet to be addressed in the family that arguably exhibits the most extreme outcomes of vertebrate social evolution, the African mole rats (Bathyergidae), leaving the ultimate causes of their many unusual adaptations open to debate. Here we report-using a 14-year field study of wild Damaraland mole rats, Fukomys damarensis - that workers appear to have strong but unusual effects on offspring. Groups with larger workforces exhibited substantially higher rates of offspring recruitment while maintaining high juvenile survival rates, relationships that may have favored the evolution of the delayed dispersal, cooperation, morphological specialization, and unusual patterns of longevity that characterize such societies. Offspring reared by larger workforces also showed slower growth, however. That reduced offspring growth in larger groups has also been documented under ad lib. food conditions in the laboratory raises the possibility that this reflects socially induced growth restraint rather than simple constraints on resource availability. Our findings shed new light on the evolution of complex sociality in this enigmatic clade and highlight further departures from the norms reported for other cooperative vertebrates.

Keywords: eusociality, cooperative breeding, helping, helper effects, group size.

\section{Introduction}

In most cooperatively breeding societies, offspring delay dispersal from their natal group and cooperate to rear future generations of the breeders' young (Solomon and French 1997; Cockburn 2004; Hughes et al. 2008). Characterizing the effect of this workforce on the fitness of breeders is central to understanding not only the evolution of delayed dispersal and cooperation (Cockburn 1998; Dickinson

\footnotetext{
* Corresponding author; e-mail: a.j.young@exeter.ac.uk.
}

Am. Nat. 2015. Vol. 186, pp. 302-311. (C) 2015 by The University of Chicago. 0003-0147/2015/18602-55985\$15.00. All rights reserved.

DOI: $10.1086 / 682048$ and Hatchwell 2004; Ekman et al. 2004) but also the origins of the derived adaptations that characterize more complex cooperative societies, such as those of the eusocial insects, including large group sizes, divisions of labor, morphological divergence between queens and workers, and unusual patterns of longevity (Keller and Genoud 1997; Bourke 1999, 2011). It is notable, therefore, that the effect of workforce size on the reproductive success of breeders has yet to be established in the one vertebrate group that exhibits all of these adaptations: the African mole rats (Jarvis and Bennett 1993; Jarvis et al. 1994; Bennett and Faulkes 2000).

The societies of naked and Damaraland mole rats (Heterocephalus glaber and Fukomys damarensis, respectively) and some of their social relatives (genera Fukomys and Cryptomys) may constitute the closest that vertebrates have come to making the evolutionary transition to eusociality (Jarvis 1981; Jarvis and Bennett 1993; Crespi and Yanega 1995; Burda et al. 2000; Boomsma 2009), exhibiting an array of adaptations more typical of the eusocial insects than other cooperative vertebrates. They live in some of the largest vertebrate families known (up to 300 individuals in naked mole rat societies; Brett 1991) and exhibit a complete division of reproductive labor (reproduction is typically monopolized by a single queen; Jarvis 1981; Jarvis and Bennett 1993), morphologically divergent queens and workers (O'Riain et al. 2000; Young and Bennett 2010), and extraordinary patterns of longevity (Sherman and Jarvis 2002; Buffenstein 2005; Dammann and Burda 2006; Dammann et al. 2011). Given strong ecological constraints on their dispersal to independent breeding positions (Jarvis et al. 1994; Faulkes et al. 1997), offspring delay dispersal from their natal groups and engage in a range of potentially mutualistic cooperative activities as workers, including the excavation of foraging tunnels, the collection of food, and antipredator defense (Jarvis and Bennett 1993; Bennett and Faulkes 2000). It has often been argued that positive effects of these workers on the reproductive success of 
breeders may have played a central role in the evolution of mole rat societies by generating (1) indirect fitness benefits that strengthened selection for delayed dispersal and cooperation (Jarvis et al. 1994; Bennett and Faulkes 2000) and (2) selection pressures that favored the morphological modification of breeders (e.g., to enhance fecundity; O'Riain et al. 2000; Young and Bennett 2010) and the elongation of life spans (e.g., increases in workforce size, and hence breeder reproductive output, with breeder age may have strengthened selection for breeder longevity; Keller and Genoud 1997; Sherman and Jarvis 2002; Buffenstein 2005). However, whether workers do have positive effects on breeder reproduction in such societies has yet to be established, given the difficulty of long-term demographic field research on these subterranean mammals (Jarvis and Bennett 1993; Jarvis et al. 1998). As such, the role of workers in the evolutionary origins of these complex societies remains open to debate.

While research on other cooperatively breeding mammals has revealed positive relationships between helper number and offspring recruitment, growth, and survival (e.g., Clutton-Brock et al. 2001; Russell et al. 2003, 2007; Hodge 2005; Sparkman et al. 2011), qualitative differences in helper behavior in mole rat societies may limit the parallels that can be drawn. While helpers in most cooperative vertebrates directly provision offspring, the role of mole rat workers in offspring provisioning is less clear (Lacey and Sherman 1991). Naked mole rat workers produce feces for consumption by pups during weaning (Jarvis 1991; Lacey and Sherman 1991), but this is less common in Damaraland mole rat societies (Cooney 1999). Young mole rats appear to transition directly from lactation to independent feeding on tubers (Jarvis 1991; Cooney 1999; Bennett and Faulkes 2000). Until offspring can tunnel effectively for themselves, however, it is likely that they are dependent on other group members for the location and exposure of tubers (Bennett and Faulkes 2000). Workers locate and expose tubers that are subsequently exploited by all group members either in situ or after being carried to a central food store close to the breeding chamber (Jarvis et al. 1998; Bennett and Faulkes 2000). The workforce could thereby enhance offspring recruitment, growth, or survival by improving resource availability and/or reducing its variance (Lovegrove 1991) for (1) the breeding female (potentially reducing interbirth intervals, increasing litter sizes, and enhancing pre- or postnatal investment per offspring; Russell et al. 2003; Russell and Lummaa 2009) and/or (2) newly weaned offspring. Mole rat workers might also enhance offspring survival directly, as they carry young away from threats and areas of active excavation, repel predators, and may also simply dilute per capita predation risk (Lacey and Sherman 1991; Bennett and Faulkes 2000).
Here we investigate whether the size of the workforce positively predicts offspring recruitment, growth, and survival in wild Damaraland mole rat societies, using data from a unique 14-year longitudinal field study. Damaraland mole rats live in extended family groups of two to 40 individuals in which the dominant female monopolizes reproduction (Burland et al. 2004) and offspring delay dispersal and contribute to a variety of cooperative activities (Jarvis and Bennett 1993; Bennett and Faulkes 2000; Burland et al. 2004). The dominant female may breed yearround (pups are found in their burrow systems throughout the year; e.g., Young et al. 2010), producing litters of one to six pups (following a gestation of 78-92 days) that suckle exclusively from her but begin to eat solid food from 6 days and are weaned by 4 weeks of age (Bennett and Jarvis 1988; Jarvis and Bennett 1993; Young et al. 2010). Damaraland mole rats live in the arid grasslands of southern Africa, where for much of the year the dry compacted-sand substrate is extremely costly to excavate (Lovegrove 1989); an ecological constraint that is thought to have favored the delayed dispersal of offspring (Jarvis et al. 1994; Faulkes et al. 1997). This constraint relaxes, however, during periods of sustained rainfall, which stimulate prolific excavation by workers of both sexes in search of food, matings, and dispersal opportunities (Jarvis and Bennett 1993; Jarvis et al. 1998; Young et al. 2010). Mole rat dispersal patterns are not well understood, but the evidence to date suggests that female Damaraland mole rats usually disperse alone to found new colonies, while males may disperse alone or in pairs, ultimately either joining such females to found new groups or immigrating in to existing colonies (Burland et al. 2004; Young et al. 2010). Whether workers have positive effects on the reproductive success of breeders is not yet known. Laboratory studies under ad lib. food conditions suggest that offspring growth rates in both Damaraland and naked mole rat societies actually decrease in the successive litters born to a breeding pair as their workforce increases in size (Bennett and $\mathrm{Na}$ varro 1997; O'Riain and Jarvis 1998), though such patterns could reflect artificial constraints in captivity (Lacey and Sherman 1991). The limited field evidence to date suggests that larger groups are more likely to be recaptured than smaller ones (Jarvis et al. 1994), but whether this reflects an effect of group size on reproductive success - rather than adult survival, dispersal, or simply the detectability of groups in the field-is unknown.

\section{Material and Methods}

\section{Study Population and Trapping Methods}

Between April 1988 and March 2002, a longitudinal field study of Damaraland mole rats was conducted at Dordabis, 
Namibia $\left(22^{\circ} 58^{\prime} \mathrm{S}, 17^{\circ} 41^{\prime} \mathrm{E}\right)$. A median (interquartile range [IQR]) of 24 (12-37) social groups was trapped per year at approximately 6-month intervals (median [IQR] interval, 6 [5-8] months) to monitor individual recruitment, survival, growth, and dispersal (for detailed field methods, see Jarvis et al. 1998). All protocols were approved by the University of Cape Town ethics committee. Briefly, groups were trapped by digging a short trench across a line of molehills to locate the underlying tunnel and then setting pipe traps baited with sweet potato. Traps were checked every 1-3 h between 0600 and 2200 hours, and any trapped animals were weighed using a spring balance or electronic scales ( $\pm 1 \mathrm{~g}$ ), transferred to a large sand-lined box for housing with the other group members, and sustained on sweet potato and local bulbs. Once the group had been completely trapped out (gauged by an absence of activity at the trap site for $36 \mathrm{~h}$ ), any unmarked animals (distinguished until now by their sex, weight, and coat patterns) were marked by minimal toe clipping (Jarvis and Bennett 1993; Jarvis et al. 1994, 1998; Burland et al. 2004), and the entire group was returned together to their original tunnel system. A group was defined as the colony of one or more individuals caught from the same contiguous burrow system. Typically, all group members were caught from a single trap site, but occasionally two distant trap sites were found to be catching from the same group, as highlighted by our knowledge of prior group compositions and/or the absence of a dominant female in one half and confirmed by the release of individuals at one trap site and their subsequent capture at the other. The dominant female in each group (of which there was never more than one) could be readily distinguished by her perforate vagina and/or swollen teats (validated by Burland et al. 2004), and the sexes were differentiated by genital morphology (Bennett and Faulkes 2000).

\section{Statistical Analyses}

Given the mole rats' subterranean ecology, longitudinal field studies comprise the repeated trapping of study groups over time. Insights regarding workforce effects must therefore be drawn from the effects of worker numbers on the rates of offspring recruitment, survival, and growth over the intervals between successive trapping sessions for the same group (here termed "monitoring intervals"). To maximize the accuracy of our recruitment and survival metrics for each interval (see below) and their temporal congruence with our worker number measures from the start and end of the interval, we have restricted our attention throughout to monitoring intervals of no more than 6 months. To ensure that our analyses were not confounded by effects of dominant female turnover (the incidence of which could be correlated with worker number), we further restricted our analyses to monitoring intervals over which the dom- inant female remained unchanged. Each of our three major analyses (the effects of worker numbers on offspring recruitment, survival, and growth) was conducted using general linear mixed models (GLMMs), with group identity fitted as a random factor to account for the inclusion of multiple monitoring intervals from each group in each analysis and, as far as possible, for effects of between-group variation in territory or breeder quality. We did not fit dominant female identify as an additional random factor, since data were available from only one dominant female per group.

To test the prediction that worker number explains significant variation in each reproductive performance metric, each model was initially fitted with worker number (see below) as a fixed effect predictor along with several other fixed effects fitted to control for other sources of variation (e.g., rainfall, juvenile sex and mass, and interval duration; see below). Model simplification was then conducted to yield a minimal adequate model, retaining only those predictors whose removal yielded a significant reduction in the explanatory power of the model. The assessments of statistical significance reported for each term are those calculated from the change in explanatory power on removal of that term from the minimal model (if it was in the minimal model) or following its inclusion in and subsequent removal from the minimal model (if it had not been retained in the minimal model). In only one case was a significant effect of worker number accompanied by a marginal $(.05<P<.10)$ effect of a covariate (the effect of sex in the growth model). In this case, we verified that the apparent effect of worker number remained, regardless of whether the covariate in question was retained or removed from the minimal model (see "Results"). All statistical tests were two tailed and conducted using GenStat 13 (Lawes Agricultural Trust, Rothamsted). The data sets for all three analyses have been deposited in the Dryad Digital Repository, http://dx.doi.org/10.5061/dryad.1366f (Young et al. 2015).

For each of the three main models, we took a two-step approach to modeling the effects of worker number. First, we fitted as a predictor our best estimate of the number of workers that were present during the focal monitoring interval, which we term "average worker number." This was calculated as the number of workers (individuals $>50 \mathrm{~g}$; see below) present at the start of each monitoring interval, averaged with the number of these workers that were still present at the end of the interval. This approach accounts for the fact that not all workers present at the start of each interval were present (and hence available to work) throughout the interval, and it ensures that none of the youngsters newly recruited during the interval contribute to this metric of worker number (which might otherwise generate false correlations between average worker number and reproductive performance). All individuals $>50 \mathrm{~g}$ at the start of the 
interval were considered workers, since such individuals forage independently and contribute to varying degrees to all activities within the group (Bennett 1989; Jarvis and Bennett 1993; Bennett and Faulkes 2000). Second, we reran the models with two other predictors substituted in place of average worker number to allow us to partition any apparent effect of average worker number into the effects of (1) the number of workers present at the start of the interval and (2) the number of these that were lost during the interval. This second approach deals with a potential complication that could arise in the first from correlated impacts of the environmental conditions during an interval on both worker retention and reproductive performance during that interval (because while average worker number over the interval will be impacted by worker retention during the interval, worker number at the start of the interval will not). To allow for the possibility that worker number has nonlinear effects, we also fitted the quadratic of average worker number (in the first approach) and the quadratic of worker number at the start of the interval (in the second approach), though neither ever had significant effects (see "Results").

Because rainfall of $>15 \mathrm{~mm}$ per week softens the compacted sand soils at the appropriate depth to facilitate mole rat burrowing (and may thereby enhance long-term access to food for all group members; Jarvis et al. 1998), we fitted as a covariate in each model the total rainfall in the year preceding the end of the focal monitoring interval, calculated using only those weeks in the year with $>15 \mathrm{~mm}$ of rain. Simply fitting the total annual rainfall in the year leading up to the end of the focal monitoring interval yielded similar results, as did fitting the mean monthly rainfall experienced during the trapping interval (there were no significant effects of any of these rainfall metrics in any of the models). In each model, we also fitted the interaction between worker numbers (average worker number in the first approach and the number of workers at the start of the interval in the second) and rainfall to allow for the possibility of environment-dependent effects of workforce size (Rubenstein 2011), though no such interactions were detected in any of our models, and so we do not consider them further. To further allow for the possibility of seasonal effects in all models, we also tested for effects of the (1) season (either winter [April-September] or summer [October-March]) and (2) month of the year in which the second trapping event of the focal trapping interval occurred. Both seasons were well represented in each data set: offspring recruitment (summer: 64 measures; winter: 63 measures), juvenile survival (summer: 25 measures; winter: 57 measures), and juvenile growth (summer: 21 measures; winter: 44 measures). However, there were no significant effects of either factor in any of the models (season: all $\chi^{2}<2.03, P>.15$; month: all $\chi^{2}<6.47, P>.39$ ), and so for brevity we do not consider them further.

\section{Effects of Workforce Size on Offspring Recruitment Rate}

Focusing our attention on groups that were retrapped within 6 months and retained the same dominant female across the interval (127 monitoring intervals over 55 groups) frequently revealed new individuals in the second trapping session that had never previously been trapped in that group. These new arrivals could reflect within-group offspring recruitment or the immigration of mature individuals from other groups. However, all individuals in our population that were known to have immigrated from other groups exceeded $100 \mathrm{~g}$ in mass when trapped in their new group, and previous research suggests that offspring are unlikely to exceed $100 \mathrm{~g}$ in mass in their first 6 months of life (Bennett and Navarro 1997; Young and Bennett 2013). As such, we considered all individuals newly trapped following monitoring intervals of less than 6 months to be offspring recruited from within the group if they weighed $\leq 100 \mathrm{~g}$. This approach should avoid the misclassification of immigrants as within-group recruits but could underestimate absolute rates of offspring recruitment. However, this is unlikely to have affected our conclusions, since repeating our analysis with recruits reclassified as all new arrivals $\leq 140 \mathrm{~g}$ that were not known to have immigrated from elsewhere (which encompasses $>95 \%$ of new arrivals) only increased the statistical significance of the worker number relationships detected.

To investigate the effects of worker numbers on offspring recruitment, we conducted the two-step modeling approach outlined above, with the rate of recruitment (number of recruits divided by interval duration in months, logarithm transformed to normalize model residuals) set as the response term in a GLMM with normal error structure. In addition to the worker number terms (outlined above), we fitted rainfall (see above) and interval duration as fixed effects. Because young dominant females could conceivably independently have fewer workers and lower fecundity (potentially generating a spurious correlation between the two), we repeated the analysis to verify whether the effects of worker number held after excluding all intervals in which the dominant female could possibly have been $<3$ years old. Because dominant females were rarely of known age, we conservatively based our exclusions on a minimum possible age for each female, calculated utilizing her mass at first capture: if she weighed $<100 \mathrm{~g}$ on first capture, she was conservatively assumed to have been 6 months old on first capture, and if she weighed $>100 \mathrm{~g}$ on first capture, she was assumed to have been just 12 months old on first capture (Young and Bennett 2013).

\section{Effects of Workforce Size on Juvenile Survival}

Individuals trapped as juveniles ( $\leq 50 \mathrm{~g}$ body mass) were never subsequently retrapped outside their natal groups 
within 6 months, suggesting that they do not disperse until after this time. Juveniles that were never retrapped after first being detected, despite their natal group being retrapped within 6 months, were therefore considered to have died rather than dispersed. To investigate the effects of worker number on juvenile survival, we conducted the two-step modeling approach outlined above, with juvenile survival (for the 82 juveniles whose 28 groups were retrapped within 6 months) set as the binomial response term in a GLMM. In addition to the worker number terms (outlined above), we fitted the following predictors as fixed effects: rainfall, interval duration, juvenile sex and mass, and number of juveniles at the start of the interval.

\section{Effects of Workforce Size on Juvenile Growth Rate}

Of the 68 juveniles that survived their first monitoring interval, all but three were reweighed when retrapped at the end of the interval, yielding a sample of 65 juveniles from 27 groups for which growth rates over their first interval could be calculated. Growth rates were calculated as the difference in mass of the juvenile between its first capture (at $\leq 50 \mathrm{~g}$ ) and its subsequent capture $<6$ months later, divided by the interval duration in months. To investigate the effects of worker number on juvenile growth rates, we conducted the two-step modeling approach outlined above, with growth rate (square root transformed to normalize model residuals) set as the response term in a GLMM with normal error structure. We fitted juvenile mass at the start of the interval and interval duration as covariates to control for the expected slowing of growth as offspring increased in mass and hence over time. In addition to the worker number terms (outlined above), we also fitted the following predictors as fixed effects: rainfall, juvenile sex, and the number of juveniles at the start of the interval.

\section{Results}

\section{Effects of Workforce Size on Offspring Recruitment Rate}

The focal groups varied markedly in size, from unassisted breeding pairs to those with 34 workers. Over 127 monitoring intervals (between successive trapping events up to 6 months apart) for 55 groups, the groups recruited between 0 and 11 offspring, with recruitment rates over the interval ranging from 0 to 2.28 offspring/month. There was a significant positive effect of average worker number over the monitoring interval on the rate of offspring recruitment during the interval $\left(\chi^{2}=4.86, P=.030\right)$. Partitioning average worker number revealed a significant positive effect of the number of workers present at the start of the interval $\left(\chi^{2}=10.09, P=.002\right.$; fig. $\left.1 A\right)$ and a significant negative effect of the number of these workers that were lost from the group during the interval $\left(\chi^{2}=8.54, P=\right.$ .004). There were no significant effects of any other variables in either model (rainfall: $\chi^{2}<0.01, P>.94$; worker number quadratic term: $\chi^{2}\langle 2.25, P>.14$; interval duration: $\chi^{2}<1.06, P>.31$ ). These associations between worker number and recruitment rate were robust to the exclusion of all intervals from young dominant females (who could conceivably have had both reduced fecundity and few workers); excluding all intervals in which the dominant female could have been $<3$ years old at the start of the interval (leaving 54 intervals from 29 groups) left the effects of worker number qualitatively unchanged both before partitioning (positive effect of average worker number: $\chi^{2}=4.80, P=$ .036 ) and afterward (positive effect of worker number at the start of the interval: $\chi^{2}=7.52, P=.010$; negative effect of the number of workers lost during the interval: $\chi^{2}=5.74$, $P=.020)$.

\section{Effects of Workforce Size on Juvenile Survival}

Of the 82 juveniles whose 28 groups were retrapped within 6 months of the juvenile's first detection, 68 survived the monitoring interval, while 14 did not (82.9\% survival). There was no significant effect of average worker number over the interval on the probability of juvenile survival through the interval $\left(\chi^{2}=2.39, P=.13\right)$. Accordingly, partitioning average worker number revealed a tendency for a positive effect of the number of workers present at the start of the interval $\left(\chi^{2}=3.06, P=.084\right.$; fig. $1 B$ ) and no significant effect of the number of these workers that were lost from the group during the interval $\left(\chi^{2}=2.00, P=.16\right)$. There were no significant effects of any other variables in either model (worker number quadratic term: $\chi^{2}<1.43$, $P>.23$; juvenile mass at first capture: $\chi^{2}=3.46, P=.063$; juvenile sex: $\chi^{2}=0.09, P=.76$; juvenile number: $\chi^{2}=$ $1.75, P=.19$; rainfall: $\chi^{2}=0.65, P=.42$; interval duration: $\left.\chi^{2}=0.73, P=.40\right)$.

\section{Effects of Workforce Size on Juvenile Growth Rate}

Of the 68 juveniles that survived their first monitoring interval, all but three were reweighed on recapture at the end of the interval, yielding a sample of 65 juveniles from 27 groups for which growth rates could be calculated over the interval. Juveniles grew at rates ranging widely from 1.96 to $20.60 \mathrm{~g} / \mathrm{month}$. There were significant negative effects of both juvenile mass at the start of the interval $\left(\chi^{2}=\right.$ 8.94, $P=.004)$ and the length of the interval $\left(\chi^{2}=17.16\right.$, $P<.001)$ on juvenile growth rates during the interval, as would be expected of a typical growth trajectory in which growth rate slows with increasing size and hence over time. 

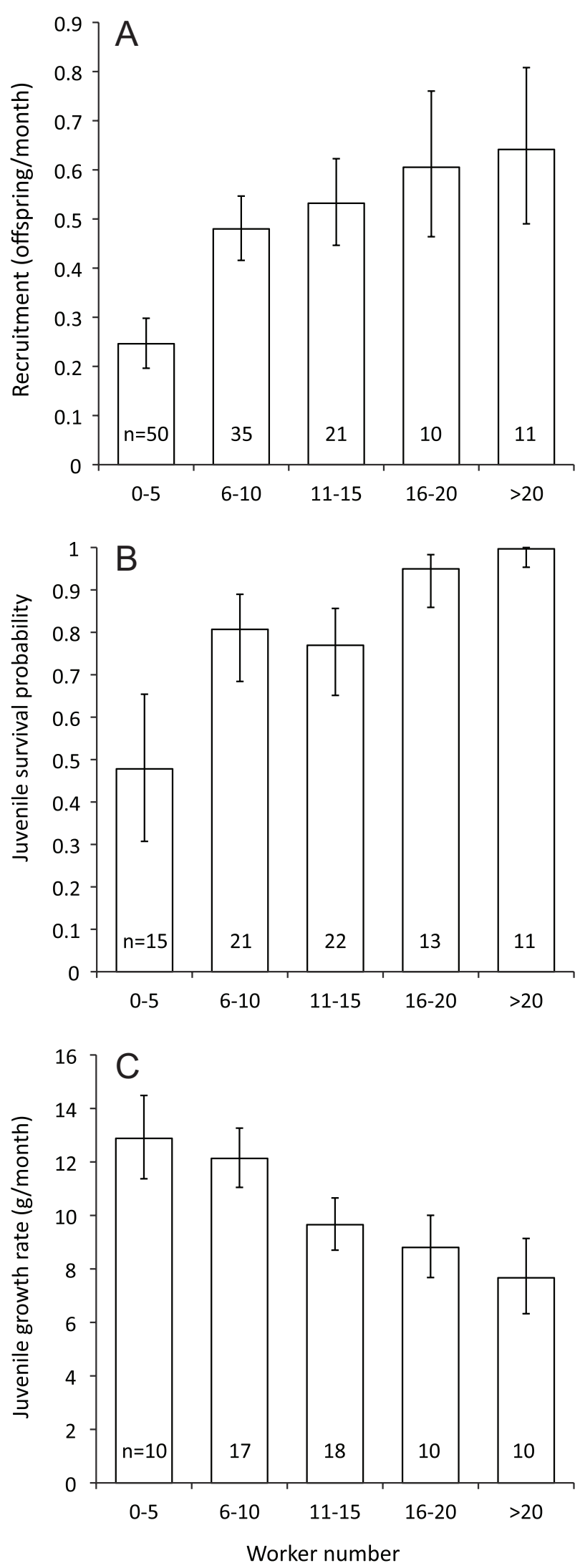

While controlling for these effects, there was a significant negative effect of average worker number over the interval on juvenile growth rates during the interval $\left(\chi^{2}=5.34, P=\right.$ .024). There was also a tendency for juvenile males to show higher growth rates than females $\left(\chi^{2}=3.85, P=.056\right)$; conservatively retaining this term in the minimal adequate model left the statistical significance of the average worker number term qualitatively unchanged $\left(\chi^{2}=6.29, P=.015\right)$. Partitioning average worker number revealed a significant negative effect of the number of workers present at the start of the interval $\left(\chi^{2}=9.13, P=.004\right.$; fig. $\left.1 C\right)$ and a significant positive effect of the number of these workers that were lost over the interval $\left(\chi^{2}=10.63, P=.002\right)$ while again controlling for the initial mass $\left(\chi^{2}=10.00, P=.002\right)$ and interval duration effects $\left(\chi^{2}=21.16, P<.001\right)$. Again, retaining sex in the minimal adequate model (given its borderline significance: now $\chi^{2}=3.81, P=.058$ ) left the significance of these worker number terms qualitatively unchanged (worker number at start: $\chi^{2}=10.27, P=.002$; worker number lost: $\left.\chi^{2}=11.18, P=.002\right)$. There were no significant effects of any other variables in either model (worker number quadratic term: $\left.\chi^{2}<0.21, P\right\rangle .65$; juvenile number: $\chi^{2}<1.73, P>.19$; rainfall: $\chi^{2}<0.92, P>.34$ ).

\section{Discussion}

The social mole rats provide some of the most extreme examples of vertebrate social evolution (Jarvis 1981; Jarvis and Bennett 1993; Crespi and Yanega 1995; Burda et al. 2000; Boomsma 2009). However, the difficulty of longitudinal field research on these species has left it unknown whether and how the workforce impacts the reproductive success of breeders and, consequently, whether beneficial effects of the workforce are likely to have played a role in the evolution of these unique societies. Our analyses suggest that the presence and/or actions of workers in Damaraland mole rat societies have a positive effect on the reproductive success of breeders, principally by promoting offspring re-

Figure 1: Number of workers present at the start of a monitoring interval had a significant positive effect on the rate of offspring recruitment during the interval $(A)$, no significant effect on juvenile survival probability (though a positive trend is apparent; $B$ ), and a significant negative effect on offspring growth rates during the interval $(C)$. Bars present predicted means and standard errors from the general linear mixed models, with worker number at the start of the monitoring interval factorized for presentation (it was fitted in all models as a continuous variable) while controlling for the effects of the number of these workers that were lost during the interval and all other significant terms in each final model (see "Results"). The sample size contributing to each bar is presented at the base of each bar; $n$ indicates trapping intervals over which recruitment was assessed $(A)$, offspring whose survival was assessed $(B)$, and offspring whose growth rate was assessed $(C)$. 
cruitment. This finding, while necessarily correlative, lends strength to the view that positive effects of workers on breeder fitness may have favored the evolution of delayed dispersal, sociality, and cooperation in these subterranean mammals (Jarvis and Bennett 1993; Jarvis et al. 1994) as well as the queen-worker dimorphisms and unusual patterns of longevity that the social mole rats share with some eusocial insects (O'Riain et al. 2000; Dammann and Burda 2006; Dengler-Crish and Catania 2007; Young and Bennett 2010; Dammann et al. 2011). Indeed, because our analyses necessarily utilize only those monitoring intervals in which the focal group persisted across the interval, they may tend to underestimate any positive impact of the workforce on offspring recruitment and survival, since nonrecruiting small colonies may be more likely to fail-and hence be absent from our data set - than nonrecruiting large colonies (Jarvis et al. 1994; 1998). Unexpectedly, and unusually for cooperative vertebrates, our findings also suggest that offspring grow more slowly when the workforce is large, the fitness implications of which will depend upon the mechanisms at play and the extent to which any growth deficit can be recovered later in life (see below). We discuss the mechanisms that may mediate these apparent effects of workforce size and their implications for the likely origins of complex sociality in this enigmatic clade.

Our findings are necessarily correlative, given the confounding complications that would arise from manipulating workforce size (Cockburn 1998; Dickinson and Hatchwell 2004), which in mole rat societies could include potentially marked effects of hierarchy disruption on the patterns of growth and labor within colonies (O’Riain and Jarvis 1998) and the generation of an artificial mismatch between group size and burrow system architecture. Indeed, to our knowledge, causal effects of helper number on offspring recruitment have yet to be demonstrated in any wild mammal, given the difficulties entailed in long-term manipulations of helper number (Clutton-Brock et al. 2001). While the causality of the observed positive relationship between workforce size and offspring recruitment is therefore open to debate, several points lend support to the case for a causal effect of workforce size. First, if spatial variation in resource availability (Jarvis et al. 1998) simply left resource-rich groups with higher offspring recruitment rates and consequently larger workforces, groups with larger workforces might also be expected to show higher offspring growth rates, given their stronger resource bases, yet we found the opposite pattern; offspring showed growth deficits in larger groups (though such growth deficits could conceivably arise in larger groups, despite a stronger resource base, through socially induced growth suppression; see below). Second, the fact that the positive association between average workforce size over a monitoring interval and offspring recruitment during that interval partitions into a positive effect of the workforce size at the start of the interval and a negative effect of the fall in workforce size during the interval, is consistent with offspring recruitment being sensitive to within-group changes in workforce size. Third, it is unlikely that our recruitment findings simply reflect young dominant females having both lower fecundity and fewer workers, since they held even after the exclusion of all data from dominant females $<3$ years of age. Moreover, while naked mole rat queens undergo fecundity-enhancing vertebral elongation as they age (O'Riain et al. 2000; DenglerCrish and Catania 2007), the limited evidence to date suggests that Damaraland mole rat queens do not, but may instead reduce their skull growth, a tactic that might also enhance fecundity (simply by releasing resources) but should not leave it steadily increasing with age (Young and Bennett 2010).

A positive effect of workforce size on offspring recruitment could have arisen through several causal mechanisms. First, by locating and exposing tubers that all group members can exploit, larger workforces may allow the dominant female to increase her reproductive output (by increasing litter size [Taborsky 1984] and/or reducing interbirth interval [Russell et al. 2003]) by increasing her access to resources (or reducing its variance; Lovegrove 1991; Jarvis et al. 1998) and/or reducing her need to forage for herself. Second, if larger workforces decrease the variance in postnatal resource availability for offspring (Lovegrove 1991), this might impact how breeders resolve an offspring size/number trade-off. Breeders with smaller workforces might benefit from producing fewer larger young, if greater prenatal investment buffered offspring against the higher variance in postnatal food availability faced in small groups. Finally, an effect of workforce size on offspring recruitment could also be due in part to impacts on the early postnatal survival of offspring (before their first capture), arising from workforce effects on early-life resource availability, thermoregulation, or predation risk (Bennett and Faulkes 2000). The relative importance of such pre- and postnatal processes might now be teased apart by using ultrasound equipment to determine in utero litter sizes (Russell et al. 2003), with a view to establishing whether mothers with larger workforces carry larger litters and whether early postnatal survival is higher in larger groups.

In several cooperative mammals, offspring reared by more helpers grow faster and may enjoy downstream fitness benefits from doing so (Hodge 2005; Clutton-Brock et al. 2006; Russell et al. 2007; Sparkman et al. 2011). By contrast, young Damaraland mole rats reared by larger workforces show reduced growth rates. These growth deficits seem unlikely to be a simple by-product of being reared in larger litters (given the higher recruitment rates of larger groups), since juvenile growth rates were unrelated to the number of juveniles present at the start of the mon- 
itoring interval. They could instead reflect resource constraints arising from foraging competition, with the larger number of mature individuals in groups with larger workforces. Dominant females in larger groups might be able to maintain higher rates of offspring production regardless, if their rank afforded them differential access to the resources made available by the workforce. That reduced offspring growth rates have also been documented in larger captive colonies of Damaraland and naked mole rats with ad lib. access to food (Bennett and Navarro 1997; O’Riain and Jarvis 1998) raises the possibility that offspring in larger groups instead exercise growth restraint or experience socially induced growth suppression. Larger individuals may suppress the growth of offspring (so as to maintain competitive advantages; Clutton-Brock et al. 2006; Young and Bennett 2013) via exclusion from key resources or by policing growth restraint with threats of punishment (Wong et al. 2007; Young 2009; Cant and Young 2013). Indeed, evidence that the removal of middle-ranking subordinate mole rats yields striking growth responses from more junior group members in naked mole rat societies is consistent with this view (O'Riain and Jarvis 1998). Ultimately, the fitness implications of these growth deficits remain unclear (1) because they will depend on the extent to which any growth deficit can be recovered later in life and the costs of doing so (Metcalfe and Monaghan 2001; Lee et al. 2013) and (2) because such patterns could also conceivably reflect costly growth acceleration in small groups (Metcalfe and Monaghan 2001) if maturing quickly yields benefits in this context.

Unlike helpers in many other cooperative vertebrates, Damaraland mole rat workers rarely provision offspring directly, leaving their cooperative behavior less clearly subject to the evolutionary paradox of altruism (Cooney 1999). Instead, they contribute to a range of cooperative behaviorsincluding locating tubers, stocking the communal food store, and maintaining and defending the burrow system - that could conceivably yield sufficient direct fitness benefits to explain their evolution in the absence of impacts on offspring. Nevertheless, our findings suggest that, in so far as the apparent effect of workers on offspring recruitment is causal and arises from cooperation per se (rather than simply worker presence), such cooperative behavior is likely to generate indirect fitness benefits by enhancing the recruitment of kin. Similarly, while delayed dispersal most likely evolved through direct benefits of philopatry arising from ecological constraints on dispersal (Jarvis et al. 1994), these direct benefits may be complemented by indirect benefits of philopatry arising from positive effects of philopatric workers on the recruitment of kin. Indeed, our findings also highlight the challenge faced by those mole rat pairs that do attempt to found colonies of their own (Burland et al. 2004), given the low mean recruitment rates that would initially be expected in the absence of a workforce.
Our findings, the first of their kind for mole rats, have implications too for understanding the origins of the more unusual traits that some of the social mole rats shareperhaps uniquely among vertebrates - with some eusocial insects. The queen-worker dimorphisms observed in naked and Damaraland mole rat societies might well reflect selection for fecundity-enhancing changes in growth patterns on dominance acquisition (O'Riain et al. 2000; Young and Bennett 2010), given the higher reproductive rates that our findings suggest are possible in the presence of a workforce. Similarly, the extraordinary life spans of naked mole rats (Sherman and Jarvis 2002) and the greater longevities of queens than workers in captive Damaraland mole rats and their congeners (Dammann and Burda 2006; Dammann et al. 2011) may well be due in part to a positive effect of the workforce on reproductive output. Because Damaraland mole rats found new groups as pairs or trios (Burland et al. 2004), a new breeder's workforce may increase in size as they age, driving an age-related increase in reproductive success that may select for longer breeder life spans (Keller and Genoud 1997). Ultimately though, the rarity of such breeder-helper disparities in morphology and life span in other cooperative vertebrates implicates a role for other factors too in their evolutionary origins. Key among these may be the occupation of a protected expandable nest site within the foraging substrate, a trait that mole rats share with termites and some ants (Alexander et al. 1991) and the consequent scope for the evolution of a rank-related division of foraging labor. By absolving established queens from the need to forage, divisions of foraging labor may have facilitated their morphological specialization and reduced their exposure to extrinsic mortality, the latter selecting in turn for the extension of breeder life spans (Keller and Genoud 1997; Young and Bennett 2010; Dammann et al. 2011). Together, our findings shed new light on the selective pressures that may have shaped the evolution of social complexity in these enigmatic mammals and highlight further departures from the norms reported for other cooperative vertebrates.

\section{Acknowledgments}

We are grateful to all those who contributed to the longterm field data, to the Lühl family for kindly allowing us to work on their property, to E. Griffin and M. Griffin for their exceptional logistical support, to the Namibian Department of Nature Conservation for permission to conduct the research, and to S. Hodge and A. Russell for comments on the manuscript and enlightening discussions. This work was funded by the National Research Foundation of South Africa and the Universities of Cape Town and Pretoria. A.J.Y. was supported by a Biotechnology and Biological Sciences Research Council David Phillips fellowship. 


\section{Literature Cited}

Alexander, R. D., K. M. Noonan, and B. J. Crespi. 1991. The evolu tion of eusociality. Pages 3-44 in P. W. Sherman, J. U. M. Jarvis, and R. D. Alexander, eds. The biology of the naked mole-rat Princeton University Press, Princeton, NJ.

$\rightarrow$ Bennett, N. C. 1989. The social structure and reproductive biology o the common mole-rat, Cryptomys hottentotus and remarks on the trends in reproduction and sociality in the family Bathyergidae. Journal of Zoology 219:45-59.

Bennett, N. C., and C. G. Faulkes. 2000. African mole-rats: ecology and eusociality. Cambridge University Press, Cambridge.

$\rightarrow$ Bennett, N. C., and J. U. M. Jarvis. 1988. The social structure and reproductive biology of colonies of the mole-rat, Cryptomys damarensis (Rodentia, Bathyergidae). Journal of Mammalogy 69:293-302.

$\rightarrow$ Bennett, N. C., and R. Navarro. 1997. Differential growth patterns between successive litters of the eusocial Damaraland mole-rat, Cryptomys damarensis, from Namibia. Journal of Zoology 241: 465-473.

$\rightarrow$ Boomsma, J. J. 2009. Lifetime monogamy and the evolution of eusociality. Philosophical Transactions of the Royal Society B: Biolog ical Sciences 364:3191-3207.

$\rightarrow$ Bourke, A. F. G. 1999. Colony size, social complexity and reproductive conflict in social insects. Journal of Evolutionary Biology 12: 245-257.

. 2011. Principles of social evolution. Oxford University Press, Oxford.

Brett, R. A. 1991. The population structure of naked mole-rat colo nies. Pages 97-136 in P. W. Sherman, J. U. M. Jarvis, and R. D. Alexander, eds. The biology of the naked mole-rat. Princeton University Press, Princeton, NJ.

$\rightarrow$ Buffenstein, R. 2005. The naked mole-rat? a new long-living model for human aging research. Journals of Gerontology A: Biological Sciences and Medical Sciences 60:1369-1377.

$\rightarrow$ Burda, H., R. L. Honeycutt, S. Begall, O. Locker-Grutjen, and A. Scharff. 2000. Are naked and common mole-rats eusocial and if so, why? Behavioral Ecology and Sociobiology 47:293-303.

$\rightarrow$ Burland, T. M., N. C. Bennett, J. U. M. Jarvis, and C. G. Faulkes. 2004. Colony structure and parentage in wild colonies of co-operatively breeding Damaraland mole-rats suggest incest avoidance alone may not maintain reproductive skew. Molecula $\rightarrow$ Ecology 13:2371-2379.

$\rightarrow$ Cant, M. A., and A. J. Young. 2013. Resolving social conflict among females without overt aggression. Philosophical Transactions of the Royal Society B: Biological Sciences 368:20130076.

$\rightarrow$ Clutton-Brock, T. H., S. J. Hodge, G. Spong, A. F. Russell, N. R. Jordan, N. C. Bennett, L. L. Sharpe, and M. B. Manser. 2006. Intra-sexual competition and sexual selection in cooperative mam mals. Nature 444:1065-1068.

$\rightarrow$ Clutton-Brock, T. H., A. F. Russell, L. L. Sharpe, P. N. M. Brotherton, G. M. McIlrath, S. White, and E. Z. Cameron. 2001. Effects of helpers on juvenile development and survival in meerkats. Science 293:2446-2449.

$\rightarrow$ Cockburn, A. 1998. Evolution of helping behavior in cooperatively breeding birds. Annual Review of Ecology and Systematics 29 $141-177$.

- 2004. Mating systems and sexual conflict. Pages 81-101 in W. D. Koenig and J. L. Dickinson, eds. Ecology and evolutior of cooperative breeding in birds. Cambridge University Press, Cambridge.
Cooney, R. 1999. Cooperative breeding and reproductive skew in the Damaraland mole-rat. PhD thesis. University of Cambridge.

Crespi, B. J., and D. Yanega. 1995. The definition of eusociality. Behavioral Ecology 6:109-115.

Dammann, P., and H. Burda. 2006. Sexual activity and reproduction delay ageing in a mammal. Current Biology 16:R117-R118.

$\rightarrow$ Dammann, P., R. Sumbera, C. Massmann, A. Scherag, and H. Burda. 2011. Extended longevity of reproductives appears to be common in Fukomys mole-rats (Rodentia, Bathyergidae). PLoS One 6:e18757.

$\rightarrow$ Dengler-Crish, C. M., and K. C. Catania. 2007. Phenotypic plasticity in female naked mole-rats after removal from reproductive suppression. Journal of Experimental Biology 210:4351-4358.

Dickinson, J. L., and B. J. Hatchwell. 2004. Fitness consequences of helping. Pages 48-66 in W. D. Koenig and J. L. Dickinson, eds. Ecology and evolution of cooperative breeding in birds. Cambridge University Press, Cambridge.

Ekman, J., J. L. Dickinson, B. J. Hatchwell, and M. Griesser. 2004. Delayed dispersal. Pages 35-47 in W. D. Koenig and J. L. Dickinson, eds. Ecology and evolution of cooperative breeding in birds. Cambridge University Press, Cambridge.

Faulkes, C. G., N. C. Bennett, M. W. Bruford, H. P. Obrien, G. H. Aguilar, and J. U. M. Jarvis. 1997. Ecological constraints drive social evolution in the African mole-rats. Proceedings of the Royal Society B: Biological Sciences 264:1619-1627.

$\rightarrow$ Hodge, S. J. 2005. Helpers benefit offspring in both the short and long-term in the cooperatively breeding banded mongoose. Proceedings of the Royal Society B: Biological Sciences 272:2479-2484.

Hughes, W. O. H., B. P. Oldroyd, M. Beekman, and F. L. W. Ratnieks. 2008. Ancestral monogamy shows kin selection is key to the evolution of eusociality. Science 320:1213-1216.

$\rightarrow$ Jarvis, J. U. M. 1981. Eusociality in a mammal: cooperative breeding in naked mole-rat colonies. Science 212:571-573.

. 1991. Reproduction of naked mole-rats. Pages 384-425 in P. W. Sherman, J. U. M. Jarvis, and R. D. Alexander, eds. The biology of the naked mole-rat. Princeton University Press, Princeton, NJ.

$\rightarrow$ Jarvis, J. U. M., and N. C. Bennett. 1993. Eusociality has evolved independently in two genera of bathyergid mole-rats - but occurs in no other subterranean mammal. Behavioral Ecology and Sociobiology 33:253-260.

Jarvis, J. U. M., N. C. Bennett, and A. C. Spinks. 1998. Food availability and foraging by wild colonies of Damaraland mole-rats (Cryptomys damarensis): implications for sociality. Oecologia (Berlin) 113:290-298.

$\rightarrow$ Jarvis, J. U. M., M. J. Oriain, N. C. Bennett, and P. W. Sherman. 1994. Mammalian eusociality: a family affair. Trends in Ecology and Evolution 9:47-51.

Keller, L., and M. Genoud. 1997. Extraordinary lifespans in ants: a test of evolutionary theories of ageing. Nature 389:958-960.

Lacey, E. A., and P. W. Sherman. 1991. Social organization of naked mole-rat colonies: evidence of divisions of labor. Pages 275-336 in P. W. Sherman, J. U. M. Jarvis, and R. D. Alexander, eds. The biology of the naked mole-rat. Princeton University Press, Princeton, NJ.

Lee, W. S., P. Monaghan, and N. B. Metcalfe. 2013. Experimental demonstration of the growth rate-lifespan trade-off. Proceedings of the Royal Society B: Biological Sciences 280:20122370.

Lovegrove, B. G. 1989. The cost of burrowing by the social mole rats (Bathyergidae) Cryptomys damarensis and Heterocephalus glaber: the role of soil moisture. Physiological Zoology 62:449-469. 

a question of risks, numbers, and costs. Behavioral Ecology and Sociobiology 28:37-45.

$\rightarrow$ Metcalfe, N. B., and P. Monaghan. 2001. Compensation for a bad start: grow now, pay later? Trends in Ecology and Evolution 16:254-260

$\rightarrow$ O'Riain, M. J., and J. U. M. Jarvis. 1998. The dynamics of growth in naked mole-rats: the effects of litter order and changes in social structure. Journal of Zoology 246:49-60.

$\rightarrow$ O’Riain, M. J., J. U. M. Jarvis, R. Alexander, R. Buffenstein, and C. Peeters. 2000. Morphological castes in a vertebrate. Proceedings of the National Academy of Sciences of the USA 97:13194-13197.

$\rightarrow$ Rubenstein, D. R. 2011. Spatiotemporal environmental variation, risk aversion, and the evolution of cooperative breeding as a bethedging strategy. Proceedings of the National Academy of Sciences of the USA 108:10816-10822.

$\rightarrow$ Russell, A. F., P. N. M. Brotherton, G. M. McIlrath, L. L. Sharpe $\rightarrow$ and T. H. Clutton-Brock. 2003. Breeding success in cooperative meerkats: effects of helper number and maternal state. Behavioral Ecology 14:486-492.

$\rightarrow$ Russell, A. F., and V. Lummaa. 2009. Maternal effects in cooperative breeders: from hymenopterans to humans. Philosophical Transactions of the Royal Society B: Biological Sciences 364:1143-1167.

$\rightarrow$ Russell, A. F., A. J. Young, G. Spong, N. R. Jordan, and T. H. Clutton-Brock. 2007. Helpers increase the reproductive potential of offspring in cooperative meerkats. Proceedings of the Royal Society B: Biological Sciences 274:513-524.

$\rightarrow$ Sherman, P. W., and J. U. M. Jarvis. 2002. Extraordinary life spans of naked mole-rats (Heterocephalus glaber). Journal of Zoology 258: 307-311.

Solomon, N. G., and J. A. French, eds. 1997. Cooperative breeding in mammals. Cambridge University Press, Cambridge.
Sparkman, A. M., J. Adams, A. Beyer, T. D. Steury, L. Waits, and D. L. Murray. 2011. Helper effects on pup lifetime fitness in the cooperatively breeding red wolf (Canis rufus). Proceedings of the Royal Society B: Biological Sciences 278:1381-1389.

Taborsky, M. 1984. Broodcare helpers in the cichlid fish Lamprologus brichardi: their costs and benefits. Animal Behavior 32: 1236-1252.

$\rightarrow$ Wong, M. Y. L., P. M. Buston, P. L. Munday, and G. P. Jones. 2007. The threat of punishment enforces peaceful cooperation and stabilizes queues in a coral-reef fish. Proceedings of the Royal Society B: Biological Sciences 274:1093-1099.

Young, A. J. 2009. The causes of physiological suppression in vertebrate societies: a synthesis. Pages 397-436 in R. Hager and C. B. Jones, eds. Reproductive skew in vertebrate societies: proximate and ultimate causes. Cambridge University Press, Cambridge.

Young, A. J., and N. C. Bennett. 2010. Morphological divergence of breeders and helpers in wild Damaraland mole-rat societies. Evolution 64:3190-3197.

$\rightarrow$. 2013. Intra-sexual selection in cooperative mammals and birds: why are females not bigger and better armed? Philosophical Transactions of the Royal Society B: Biological Sciences 368:20130075.

Young, A. J., J. U. M. Jarvis, J. Barnaville, and N. C. Bennett. 2015. Data from: Workforce effects and the evolution of complex sociality in wild Damaraland mole rats. American Naturalist, Dryad Digital Repository, http://dx.doi.org/10.5061/dryad.1366f.

$\rightarrow$ Young, A. J., M. K. Oosthuizen, H. Lutermann, and N. C. Bennett. 2010. Physiological suppression eases in Damaraland mole-rat societies when ecological constraints on dispersal are relaxed. Hormones and Behavior 57:177-183.

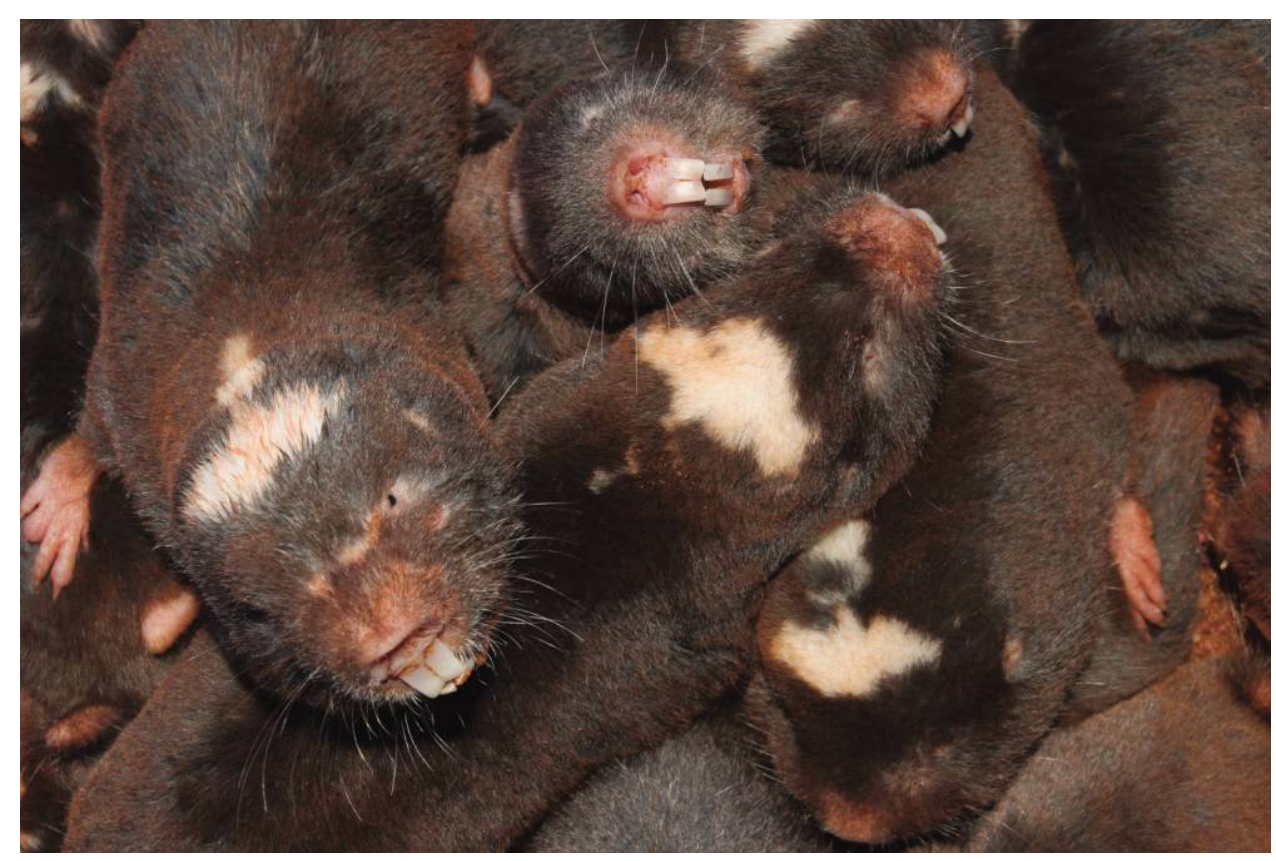

Colonies of Damaraland mole rats (Fukomys damarensis) exhibit one of the most extreme forms of vertebrate social organization, similar to that of the ants and termites. Research on wild populations has shed new light on the impact of the nonbreeding workforce on the breeding success of their queen. Photo (C) Andy Young (www.wildimages.org). 